\title{
Stanford type A aortic dissection in a patient with Marfan syndrome during pregnancy -a case report-
}

\author{
Won Ho Kim ${ }^{1}$, Jisue Bae ${ }^{1}$, Seung Won Choi ${ }^{2}$, Jong-Hwan Lee ${ }^{2}$, \\ Chung Su Kim², Hyun Sung Cho ${ }^{2}$, and Sangmin M. Lee ${ }^{2}$ \\ Department of Anesthesiolgy and Pain Medicine, ${ }^{1}$ Samsung Changwon Hospital, Sungkyunkwan University School \\ of Medicine, Changwon, ${ }^{2}$ Samsung Medical Center, Sungkyunkwan University School of Medicine, Seoul, Korea
}

\begin{abstract}
Aortic dissection during pregnancy is a devastating event for both the pregnant woman and the baby. We report a case of acute aortic dissection (Stanford type A) in a pregnant woman with Marfan syndrome at the $29^{\text {th }}$ week of gestation. She underwent a cesarean section followed by an ascending aorta and total arch replacement with cardiopulmonary bypass, without a prior sternotomy. The hemodynamic parameters were kept stable during the cesarean section by using inotropes and vasopressors under transesophageal echocardiography monitoring. The newborn survived after endotracheal intubation and management in a neonatal intensive care unit.
\end{abstract}

Key Words: Aortic dissection, Echocardiography, Marfan syndrome, Pregnancy.

There have been several case reports of successful management of aortic dissection in pregnant women with a cesarean section followed by emergency aortic surgery for a Stanford type A dissection $[1,2]$. In the previous reports, a sternotomy was performed prior to the cesarean section to facilitate urgent cardiopulmonary bypass (CPB) for possible hemodynamic instability during the cesarean section [1]. However, in our case a

Corresponding author: Won Ho Kim, M.D.

Department of Anesthesiology and Pain Medicine, Samsung Changwon Hospital, Sungkyunkwan University School of Medicine, 158, Paryongro, Masanhoewon-gu, Changwon 51353, Korea

Tel: 82-55-290-6074, Fax: 82-55-290-6578

E-mail: wonhokim.ane@gmail.com

ORCID: http://orcid.org/0000-0003-1748-1296

Received: March 13, 2014.

Revised: 1st, May 13, 2014; 2nd, May 15, 2014.

Accepted: May 16, 2014.

Korean J Anesthesiol 2016 February 69(1): 76-79

http://dx.doi.org/10.4097/kjae.2016.69.1.76 cesarean section was performed safely to deliver a baby and was followed by aortic surgery with $\mathrm{CPB}$, without a prior sternotomy. The thoracic surgical team was ready, and the $\mathrm{CPB}$ was prepared in the operating room before the cesarean section. Herein, we report this case together with a review of the literature.

\section{Case Report}

Informed consent to publish this case report was obtained from the patient. A 31-year-old woman (gravida 1, para 0) with a gestational age of $29^{+1}$ weeks was referred to our emergency room due to an acute onset of chest pain. She was $173 \mathrm{~cm}$ tall and weighed $63 \mathrm{~kg}$. The echocardiography and CT angiography revealed an acute aortic dissection (Stanford type A, DeBakey type 1) with severe aortic regurgitation. Her NT-proBNP $(885.9 \mathrm{pg} / \mathrm{ml})$ and $\mathrm{D}$-dimer $(0.79 \mu \mathrm{g} / \mathrm{ml})$ levels were elevated. The patient had no history of vascular events, hypertension, or previous vascular surgery. She presented a clinical feature of suspected Marfan syndrome and her family history included the sudden death of her father. She was admitted to the intensive

(c) This is an open-access article distributed under the terms of the Creative Commons Attribution Non-Commercial License (http://creativecommons.org/ licenses/by-nc/4.0/), which permits unrestricted non-commercial use, distribution, and reproduction in any medium, provided the original work is properly cited. 
care unit for close hemodynamic monitoring of both the mother and the fetus. Her vital signs were within the normal range at admission (systolic blood pressure: $120-150 \mathrm{mmHg}$; heart rate: $70-80$ beats/min). The pediatrician reported that the fetus' vital signs were stable and would be viable for extra-uterine life under adequate intensive care with surfactant therapy.

A cardiac anesthesiologist, thoracic surgeon, obstetrician and pediatrician discussed the treatment plan and concluded that a cesarean section followed by an ascending aorta and aortic arch replacement should be performed. The thoracic surgeon and anesthesiologist decided not to perform a sternotomy prior to the cesarean section after weighing the risks and benefits of a sternotomy before the cesarean section. Because the chest pain had developed two days prior to her visit to our ER and the patient's hemodynamic status was stable, the risk of spontaneous rupture during the cesarean section was not considered to be high. We also considered that the hemodynamic response and surgical incision for a sternotomy could only increase the risk of aortic rupture, and a delayed cesarean section due to a sternotomy could jeopardize the fetus' life. Instead, the thoracic surgical team was prepared for $\mathrm{CPB}$ in the operating room during the cesarean section.

An emergency cesarean section was performed under general anesthesia. The right radial and right femoral arterial blood pressure and central venous pressure were monitored. Labetalol $15 \mathrm{mg} / \mathrm{h}$ and nitroprusside $1 \mu \mathrm{g} / \mathrm{kg} / \mathrm{min}$ were infused before surgery. A total intravenous anesthesia with propofol and remifentanil was performed with monitoring by transesophageal echocardiography (TEE) and cerebral oximeter monitoring
(INOVOS5100B, Troy, USA). A large bore central venous line (9 Fr. Advanced Venous Access Catheter, Edwards Lifesciences LLC, Irvine, CA, USA) was accessed and connected with a rapid volume infuser (Level 1, Level 1 Technologies, Rockland, MA, USA). The intraoperative TEE revealed severe aortic regurgitation and intimal flap in the ascending aorta (Fig. 1). Cardiac output monitoring by a Swan-Ganz catheter was not performed so as not to delay the cesarean section and because a possible arrhythmia during the catheter insertion could result in hemodynamic instability. Instead, the left ventricular systolic function, the cardiac output and the degree of aortic regurgitation were monitored by TEE during the cesarean section. The hemodynamic goal during the cesarean section was to avoid an abrupt increase in blood pressure and to maintain an adequate preload and cardiac output. Considering the severe aortic regurgitation and dissection, the heart rate was controlled between 60 and 80/ $\min$.

A 1280 gm male baby was delivered with a 1 min Apgar score of 2 and resuscitated with an endotracheal intubation. The baby was admitted to the neonatal intensive care unit (ICU) and treated with a surfactant. During the cesarean section, the hemodynamic parameters were kept stable with the inotropic support of dopamine $(5-10 \mu \mathrm{g} / \mathrm{kg} / \mathrm{min})$ and norepinephrine $(0.1-0.4 \mu \mathrm{g} / \mathrm{kg} / \mathrm{min})$ and a transfusion of packed red blood cell 2 units. Colloid $500 \mathrm{ml}$ (Volulyte, Fresenius Kabi, Bad Homburg, Germany) was administered during the cesarean section.

After the cesarean section, an ascending aorta and total arch replacement, valve sparing aortic root replacement, aortic valvuloplasty with commissuroplasty, and a free graft from the aorta
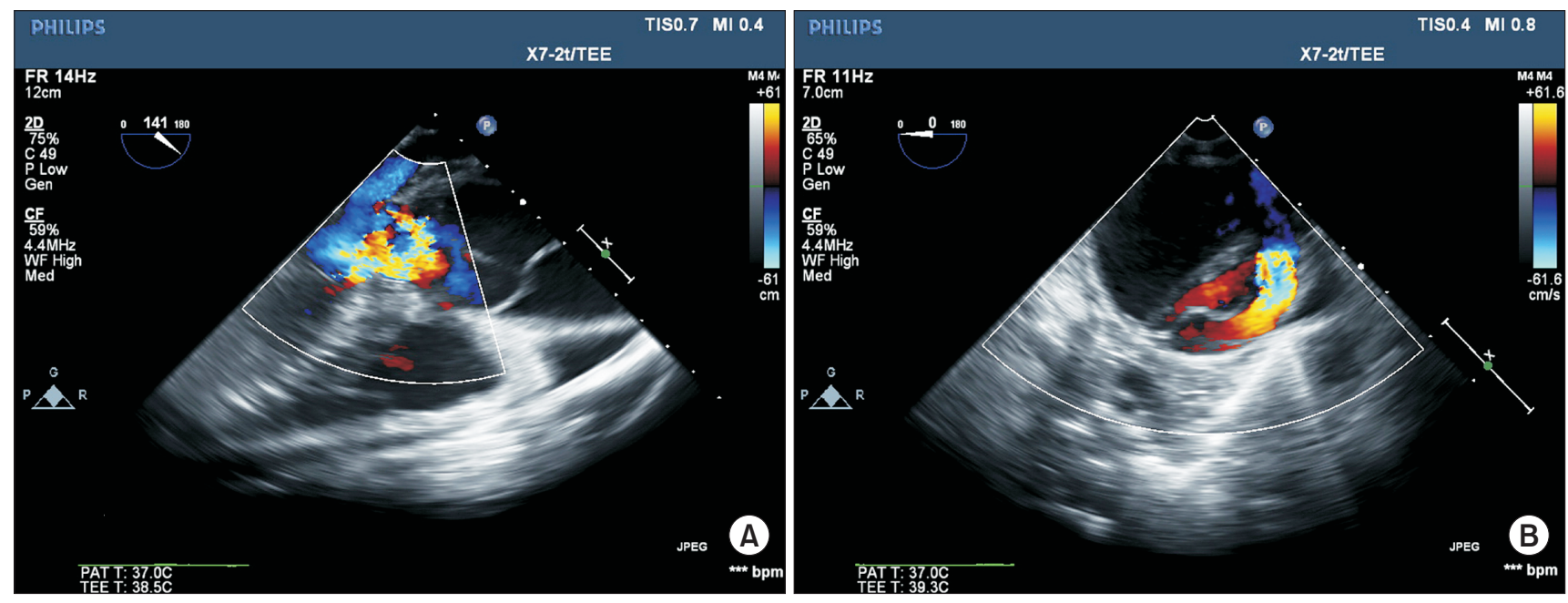

Fig. 1. (A) Transesophageal echocardiographic midesophageal long-axis images of the aortic valve showing the aneurysmal dilatation of the aortic root and ascending aorta and intimal dissection flap in the ascending aorta. Doppler color-flow imaging reveals severe aortic regurgitation caused by the outward tethering of the aortic valve cusps by aortic root dilatation. (B) Midesophageal short-axis view of the descending thoracic aorta shows an intimal dissection flap. This view confirms that the extent of this type A dissection is compatible with a DeBakey type 1 aortic dissection. 
to the right coronary artery were performed under CPB. Weaning from $\mathrm{CPB}$ was successful with dopamine $(3 \mu \mathrm{g} / \mathrm{kg} / \mathrm{min})$, dobutamine $(3 \mu \mathrm{g} / \mathrm{kg} / \mathrm{min})$, and nitroglycerine $(1 \mu \mathrm{g} / \mathrm{kg} / \mathrm{min})$. A transfusion of pRBC 2 units, cryoprecipitate 10 units, and platelet concentrate 16 units was guided by thromboelastography. The pathologic findings showed a myxoid cystic degeneration which was consistent with Marfan syndrome. The patient was transferred to the cardiac ICU and discharged without any complications on postoperative day \#6. The baby was discharged without any complications 8 weeks later.

\section{Discussion}

Aortic dissection during pregnancy is a rare condition that is life-threatening for both the pregnant woman and the baby, with a survival rate of only $20 \%-30 \%$ [3]. It is often associated with a genetic or anatomic predisposition including Marfan syndrome or bicuspid aortic valve. Marfan syndrome has been reported in up to $50 \%$ of the aortic dissections during pregnancy, with emergency surgery frequently performed at diagnosis [3-5]. The incidence of dissection during pregnancy in Marfan syndrome patients was reported to be $3 \%$ [6]. It mostly occurs during the third trimester of pregnancy due to the hyperdynamic state and hormonal effects on the vasculature [7]. Eighty percent of the dissections during pregnancy are a type A dissection, and 87.5\% of these cases occur in the pre-partum period [1,5].

Mutation in the fibrillin-1 gene is the main pathology in the Marfan syndrome. This results in a connective tissue disorder which often affects the cardiovascular system and may cause aortic root dilatation and aortic dissection [8]. Aortic root dilatation and the rapidity of this dilatation were reported to be critical risk factors for aortic dissection during pregnancy. Aortic dissection in the peripartum period mostly occurs during the third trimester, which is a period of maximal hemodynamic stress. Estrogen was reported to inhibit collagen and elastin deposition in the aorta while progesterone promotes the deposition of noncollagen proteins in the aorta $[9,10]$. When these hormonal effects occur in a patient with Marfan syndrome, aortic dissection could be initiated during pregnancy.

Previous case series have reported the successful management of aortic dissection in pregnant women through a cesarean section with subsequent emergency aortic surgery for a Stanford type A dissection $[1,10]$ or by conservative management for a Stanford type B dissection $[7,11]$. In previous case series, the sternotomy was performed prior to the cesarean section to facilitate urgent CPB for possible hemodynamic instability during the cesarean section [1]. However, in our case, the cesarean section was performed without a prior sternotomy. As mentioned previously, the onset of dissection was two days before the sur- gery, and the risk of rupture during the cesarean section was not considered to be high.

In the case of an acute type $\mathrm{B}$ dissection, minimizing the surgical repair of the dissection is recommended for a favorable outcome [7]. The medical management consists mainly of blood pressure control. Beta-blockers, calcium channel blockers and vasodilators such as sodium nitroprusside are commonly-used an anti-hypertensive agents. Vasodilators should not be used alone due to reflex tachycardia. For a type A dissection before the $30^{\text {th }}$ week of gestation, aortic repair with the fetus in utero is recommended depending on the viability of the fetus $[10,12]$. Although a high-flow, high pressure normothermic perfusion has been considered to be the safest for the fetus, there have been several case reports of successful hypothermia or deep hypothermic circulatory arrest during pregnancy before the $28^{\text {th }}$ week of gestation. In our case, the cesarean section was performed before the aortic surgery, although before the $30^{\text {th }}$ week of gestation. A decision based on the pediatrician's opinion was made that the gestational age was near 30 weeks, and the fetus was mature enough for extra-uterine life.

Aortic dissection could occur during pregnancy in patients with Marfan syndrome. To prevent this potential catastrophic event, preconception counseling should recommend prepregnancy elective aortic root replacement surgery in Marfan syndrome patients with an aortic root enlargement. Elective replacement with valve-sparing procedures and a homograft can be performed with low morbidity and mortality in patients with Marfan syndrome [13,14].

We performed general anesthesia for the cesarean section in our Marfan syndrome patient with aortic dissection during pregnancy. We chose general anesthesia because neuraxial anesthesia could produce a sympathetic block which would cause hemodynamic instability in this patient treated with a betablocker and vasodilator. However, anesthesia for a cesarean section in Marfan syndrome patients without aortic dissection would be different. A previous article recommended performing a regional anesthesia for the cesarean section so as to avoid high blood pressure which could induce aortic dissection [10]. Aortic root replacement should follow a few days after the delivery.

In conclusion, aortic dissection can occur during pregnancy in patients with Marfan syndrome, mainly in the third trimester. If possible, surgical repair of the enlarged aortic root should be performed prepartum. However, if aortic dissection Stanford type A occurs during pregnancy in a Marfan syndrome patient, a cesarean section followed by aortic surgery is currently the best modality for treatment. Careful hemodynamic management during the cesarean section under general anesthesia with TEE monitoring ensured the safety of the patient and baby without a prior sternotomy in our case. 


\section{References}

1. Ch'ng SL, Cochrane AD, Goldstein J, Smith JA. Stanford type a aortic dissection in pregnancy: a diagnostic and management challenge. Heart Lung Circ 2013; 22: 12-8.

2. Yuan SM. Aortic dissection during pregnancy: a difficult clinical scenario. Clin Cardiol 2013; 36: 576-84.

3. Nasiell J, Norman M, Lindqvist PG, Malmstedt J, Bottinga R, Blennow M. Aortic dissection in pregnancy: a life-threatening disease and a diagnosis of worth considering. Acta Obstet Gynecol Scand 2009; 88: 1167-70.

4. Zeebregts CJ, Schepens MA, Hameeteman TM, Morshuis WJ, de la Riviere AB. Acute aortic dissection complicating pregnancy. Ann Thorac Surg 1997; 64: 1345-8.

5. Rutherford RB, Nolte JE. Aortic and other arterial dissections associated with pregnancy. Semin Vasc Surg 1995; 8: 299-305.

6. Pyeritz RE. Maternal and fetal complications of pregnancy in the Marfan syndrome. Am J Med 1981; 71: 784-90.

7. Chang CY, Yang JM, Lam CW, Chen PH. Successful management of aortic dissection in a patient with Marfan syndrome during pregnancy. Am J Obstet Gynecol 2013; 208: e3-6.

8. Dean JC. Marfan syndrome: clinical diagnosis and management. Eur J Hum Genet 2007; 15: 724-33.

9. Manalo-Estrella P, Barker AE. Histopathologic findings in human aortic media associated with pregnancy. Arch Pathol 1967; 83: 336-41.

10. Immer FF, Bansi AG, Immer-Bansi AS, McDougall J, Zehr KJ, Schaff HV, et al. Aortic dissection in pregnancy: analysis of risk factors and outcome. Ann Thorac Surg 2003; 76: 309-14.

11. Katsuragi S, Neki R, Yoshimatsu J, Ikeda T, Morisaki H, Morisaki T. Acute aortic dissection (Stanford type B) during pregnancy. J Perinatol 2013; 33: 484-5.

12. Becker RM. Intracardiac surgery in pregnant women. Ann Thorac Surg 1983; 36: 453-8.

13. David TE, Armstrong S, Ivanov J, Feindel CM, Omran A, Webb G. Results of aortic valve-sparing operations. J Thorac Cardiovasc Surg 2001; 122: 39-46.

14. Gott VL, Cameron DE, Alejo DE, Greene PS, Shake JG, Caparrelli DJ, et al. Aortic root replacement in 271 Marfan patients: a 24 -year experience. Ann Thorac Surg 2002; 73: 438-43. 